\title{
Modern interpretation of FengShui in contemporary sustainable residential design
}

\author{
Z. Zhong \& B. Ceranic \\ Faculty of Arts, Design and Technology, University of Derby, UK
}

\begin{abstract}
The FengShui practice, through its core philosophy, has for centuries embraced sustainability in the design of traditional living environments and natural settlements in China. There are many encounters of sustainable FengShui manifestations in the ancient Chinese settlements, some of which have been studied in this research and interpreted from the theoretical, environmental, ecological, socio-cultural and economic perspectives. Based on the research findings and their contemporary interpretation, the link between the research and practice has been formed via undertaking the residential development design study on location in Shanghai. The results of the study thus far are reported in this paper in terms of their urban and design resolution, focusing on the pursuance of the balance between three main aspects - energy, environment and ecology. The unification between circulation of Qi and environmental elements is reflected in its modern context and represented in terms of master planning on the macro scale and consideration of shelter environmental qualities on the micro scale.
\end{abstract}

Keywords: FengShui, Qi, balance, sustainable design, environment, energy.

\section{Introduction}

FengShui (国水) is an ancient Chinese practice for achieving harmony and balance with one's environment; in choosing a place to live, siting a grave, planning a farm, even arranging furniture in a room. In Chinese, the literal meaning of 'Feng' is wind and of 'Shui' is water; water and wind being two mediums carrying and dispersing $Q i$. The actual name originates from The Burial Book written by Guo, Pu (276 - 324), but the rudimental principles of FengShui practice can be traced back as early as Zhou Dynasty and later Qin Dynasty [2]. 
The earliest written record of FengShui dates back to the Han dynasty, around AD25, but it is believed to have been part of Chinese thinking for many centuries before. Influenced by Taoism, Confucianism and Buddhism, FengShui embodies distinctive characteristics with the relationship between humans and nature being at the core of its philosophy and the practice. Whether it is the Confucian idea of man and nature becoming one, the Taoism view of the Tao reflecting nature or the Buddhist belief that all living things are equal, the philosophy was through the centuries enriched with ecological wisdom that has helped Chinese culture and civilisation to survive for thousands of years [10].

In recent years, FengShui has experienced remarkable renaissance and has even made it onto the curriculum of some secondary schools and Universities. There are in excess of 1,000 practitioners in Shanghai alone, offering their consultancy for both commercial and residential developments. During the design of Hong Kong and Shanghai Bank Headquarters (1979-1986), Foster and Partners worked in collaboration with FengShui geomancer, having to adapt to a different client beliefs and subsequent commercial reality. For example, the skewed angles of escalators were designed to keep good $Q i$ in building and to confuse the evil spirits who might try to enter. A perfectly serviceable walkway in Central district of Hong Kong was torn down after the construction because the angle at which it traversed the road was bad for $Q i$ in terms of its movement. Walt Disney consulted FengShui experts when building Disney Hong Kong, resulting in, amongst other recommendations, having to shift the main entrance by 12 degrees to ensure maximum prosperity. There are many other precedents, mostly in recent residential design where the revival is most powerful. It is thought that even the Chinese government is quietly encouraging it, worried about the people rush for the material wealth that could leave them with spiritual hole in their lives. In fact, FengShui never really went away, having to subside during the years of oppression by Communist regime but strongly resurfacing in the recent times.

\section{Philosophical principles of traditional FengShui}

At its core, the philosophy of FengShui embodies holism, circulation, balance and transformation, interpreted by the concept of Qi and the theory of Yin Yang and the Five Elements.

\subsection{Holism - the concept of Qi}

The concept of Qi is the fundamental premise of FengShui theory and largely the basis of Taoism and traditional Chinese philosophy [3]. According to FengShui, Qi is an invisible and intangible substance, a form of energy, solely responsible for providing the 'breath of life' to everything that exists in the Universe. It is believed that every human being is a type of Qi filled with subtle energy and that the natural environment comes into being through the constant movement and transformation of Qi. Through the concept of Qi, FengShui creates inseparable and unified connection between the humans, shelters and nature, emphasising the uppermost principle of pursuing and keeping the balance and harmony. 


\subsection{Circulation and balance - the theory of Yin Yang}

Based on the concept of Qi, FengShui believes the world and everything on it is formed by the circulation and interaction of Yin Qi and Yang Qi - two opposite energies that are interdependent of each other and in the constant state of the dynamic balance. Confucius $(551-479$ BC) defined this philosophy of balance by saying 'With neutrality, heaven and earth separates and all things form' [4]. Ancient Chinese further interpreted the process of circulation of Yin Qi and Yang $\mathrm{Qi}$, describing that 'when a thing reaches one extreme, it reverts from it, that is to say, extremes produce opposite reactions and each object or situation invariably gives birth to its opposite' [5]. This indicates the nucleus of Yin and Yang complementary relationship, where the neutrality and balance are the final outcome of even most opposite reactions.

Through the relationship between the opposition and collaboration, the theory of Yin Yang not only demonstrates the concept of circulation, but also reflects harmonious and balanced relationship between the humans and natural environment.

\subsection{Transformation - the theory of five elements}

Ancient Chinese perceived the whole of Universe as a dynamic world, believing that the transformation of every object on the Earth must comply with the law of life cycle and that the destruction of one thing will inevitably result in the creation of other. Hence, FengShui poses the theory of Five Elements, believing that the world is consisted of five primary elements; water, wood, fire, soil and metal. The movement and circulation of these elements explains interrelation, circulation, generative principles and orders, suggesting the interdependent and opposite relationship of all things. Combined with Qi and Yin Yang, it further explains the transformation of substance and energy in FengShui theory.

\section{Pursuance of Qi in traditional FengShui practice}

FengShui believes that the living $Q i$ is beneficial and equally that the dying $Q i$ is detrimental to human health and prosperity. Although $Q i$ is invisible and impalpable energy, people were still able to see its 'direct' appearance through many manifestations on the Earth. As the force of the Universe, Qi is integrated with the Earth, flowing on the surface known as 'water', moving along underground passages known as 'dragon veins', and growing with trees and vegetations known as 'fur of the Earth'. Six basic conditions were established by ancient Chinese for a site that constituted an 'ideal' living environment; falling back upon Dragon (ancestral, family and principal mountains), surrounding hills, nearby water, site orientation, prospering forest and requirements for a man made landscape and architectural interventions. The purpose of later was to provide shelter and remedy landscape shortcomings so to improve the accumulation of good $Q i$, prevent its dispersion and minimise the invasion of detrimental $Q i$. The central objectives was to find a suitable settlement location by investigating the landscape configuration and topography, 
means of controlling the movement of $Q i$, water supply and location, prevailing winds and surrounding trees in order to accumulate and retain good $Q i$.

From the architectural perspective, traditional FengShui environmental model was characterised by the "spatial enclosure and separation" [6]. According to Kaplan and Kaplan [7], this concept delivered privacy, security, defence and protection to the community, providing good conditions to set up a stable living environment, without great concerns about the negative outside influences.

\section{Modern interpretation - residential development study}

\subsection{Holistic design approach}

Inspired by traditional FengShui practice, a contemporary sustainable design approach should combine all the information of surrounding contextures and local environmental conditions with a thorough assessment and analysis of their impact on the various aspects of master planning, urban space resolution, residential blocks layout, building form and materials, placement, orientation and fenestration. But, perhaps more significantly, the contemporary interpretation and contextualisation of following issues established in this research is needed.

From the environmental and ecological perspective, FengShui emphasises the appropriate exploitation of natural resources, sensitive use of energy, recycling and reuse of materials, and consistent protection of the natural environment. From the socio-cultural perspective, FengShui promotes a positive individual contribution in terms of personal responsibility, respect, care, share and involvement with both family and the local community. From the socioeconomic perspective, the FengShui encourages autarkic mode of production, prudent consumption and the strong self-resumable function in relation to the local ecological system so that the balance is achieved between the production, goods consumption and exploitation of nature.

Thus, the philosophical interpretation of sustainability in traditional FengShui is based on the balance and harmony between those social, cultural, environmental, ecological and economic aspects, all of which are also important to the contemporary sustainable development.

\subsection{Contextual research and master planning}

Following the principles discussed above, the link between the research and practice has been established by undertaking the residential development design study in Gaoqiao Town, on the north east edge of the Shanghai city centre. Although this part of the research is on the early development stage, the brief analysis, design intent and site survey is completed, in terms of the site approaches, relation to immediate architecture/buildings, focal points, site entrances, site cartography, orientation, public transport services, previous site uses, trees/wildlife observations etc. Further site analysis was conducted by considering environmental, pedestrian, vehicular access, transportation infrastructure, existing green spaces, noise levels, primary traffic (see Figure 1). 


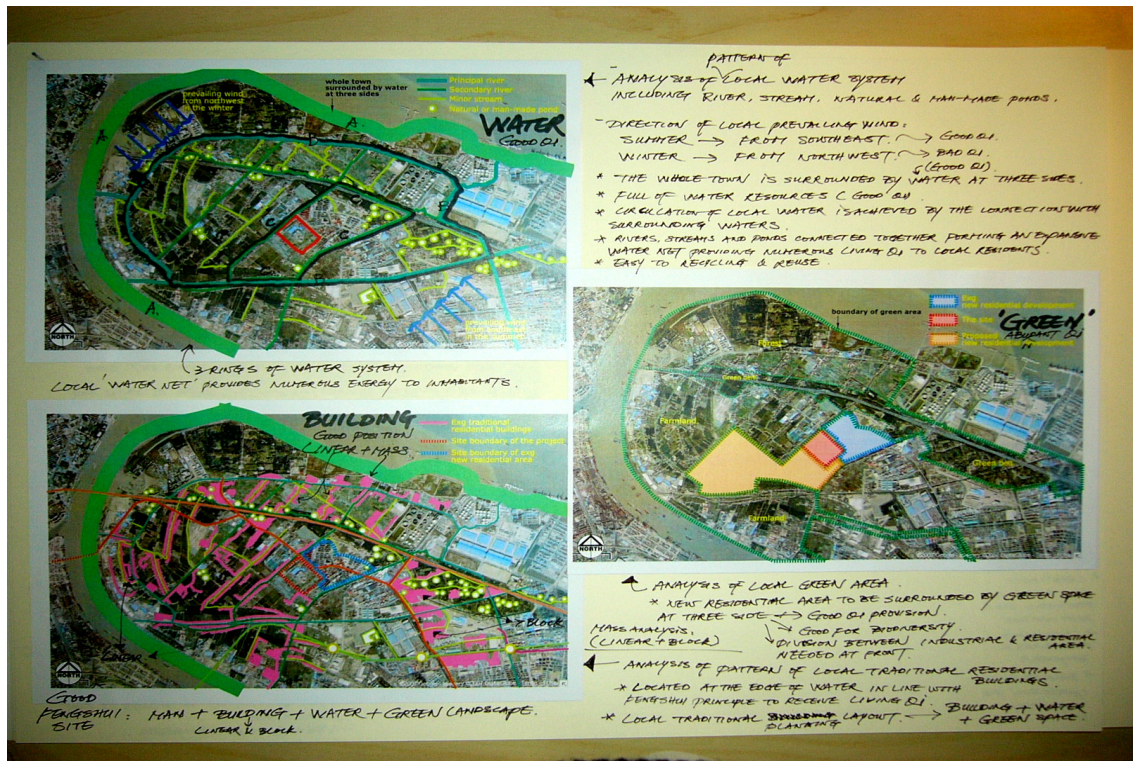

Figure 1: $\quad$ Example of site analysis - Gaoqiao town residential development.

\subsection{Movement of Qi}

Initial brainstorming on the contemporary interpretation of traditional FengShui scaled to a single residential dwelling was also undertaken. Different aspects of energy use (operational, embodied and transport), materials, water supply and conservation, waste and biodiversity were considered. Interesting parallels were discovered between the current practice in environmental building design and traditional FengShui doctrine (see Figure 2).

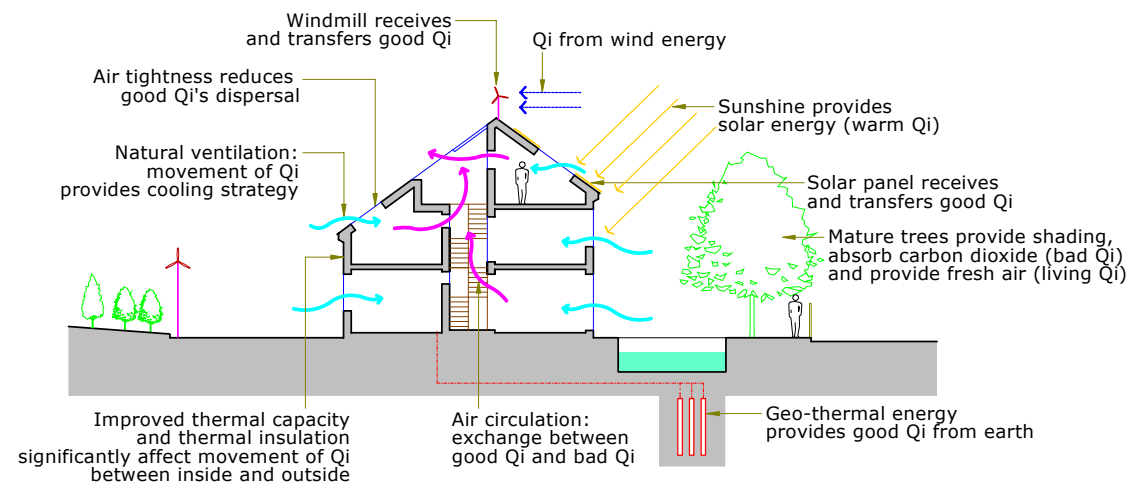

Figure 2: $\quad$ Interpretation of Qi in modern residential design context.

Whether it is use of sun energy to accumulate good Qi in the winter or use of the prevailing wind to disperse it, whilst providing cooling strategy for a house 
in summer, these are just examples of initial observations drawn between Qi movement and "usual suspects" in terms of the natural ventilation and passive solar design principles. In addition, appropriate building technology and use of high thermal mass materials further maintains good Qi inside a house, whilst improving air tightness and thermal comfort. Current research is concentrated on the development of more innovative and creative environmental strategies that are both true to FengShui principles and effective for contemporary residential design.

\subsection{Energy consumption}

The FengShui concept of balance can be used to address the energy conservation. Solar and wind energy are interpreted as good Qi and are also the most recognised renewable energy sources. By harvesting and transforming it into the operational energy the fundamental principles of circulation and transformation of Qi are achieved, whilst the balance between exploitation and consumption of renewable and non-renewable energy is improved. In addition to solar and wind energy, geo-thermal energy is a living Qi stored and generated from the earth. FengShui believes that this type of Qi has special qualities that can improve dwellers' health. Geo-thermal technologies are increasingly more exploited as a source of renewable energy that provide for both warming and cooling strategies.

\subsection{Use of local materials and waste}

FengShui believes that a good living environment is one with the right balance between Qi of human and Qi of all types of substances and materials found in the immediate surroundings (see Figure 3).

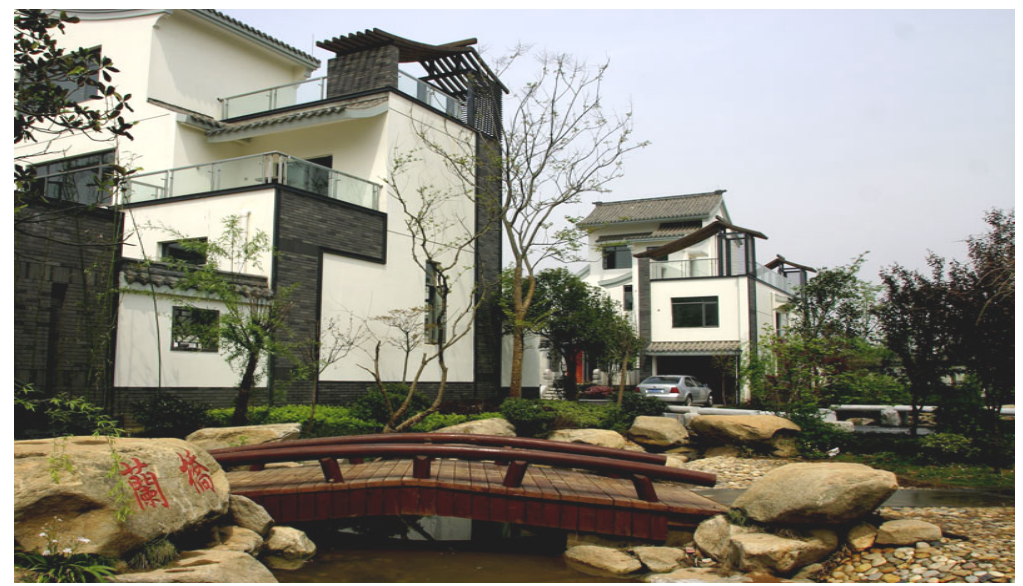

Figure 3: Good combination of Qi of different locally sourced materials in a newly developed residential area in city of Wuhan, central China. 
Thus, using and re-using the local materials for buildings and construction is the natural course in FengShui, following the fundamental principle that the destruction of one thing will inevitably result in the creation of other. In addition, local supply of other materials such as the agricultural and timber production waste is often used for heating. Generated ash provides nourishment to farm land and is favoured by local inhabitants, thus closing the life cycle discussed in the theory of five elements.

According to Wang [8], inhabitants would often use local construction waste to reconfigure the landscape and remedy its shortcomings in line with FengShui principles. 'Turning waste into a resource' is a traditional notion of Chinese FengShui, elaborated in two aspects. Firstly, it encourages positive attitude in dealing with the waste and secondly it reminds people of need to consider the whole life cycle of materials and create solutions for recycling and reuse, thus minimising the impact on the local environment.

\subsection{Use of water resources}

In FengShui, water is regarded as a rich source of living Qi and a point at which the Qi dispersed by the wind is retained. A good FengShui site is a place where living Qi exists and accumulates, where wind has its way and water has its course. In traditional FengShui settlement, FengShui trees and architectural interventions such as pagodas, pavilions, memorial gates and bridges are placed in central positions that overlook and guard local water resources. In general, the concept of circulation and balance of living Qi in FengShui practice encourages the creative use of water resources at the local level, whilst bringing the benefits to physical, psychological and economical aspects of human living. Through the thoughtful arrangements of accumulation and delivery of water flows, the modern sustainable residential development should aim to not only bring living Qi to each dwelling but also create conditions for conservation, harvesting and reuse of water resources (see Figure 4).

Through reforestation like planting trees at the edge of rivers and lakes, the accumulation of water resources and control of the movement of living Qi is achieved. FengShui encourages collection and storage of water. Apart from river

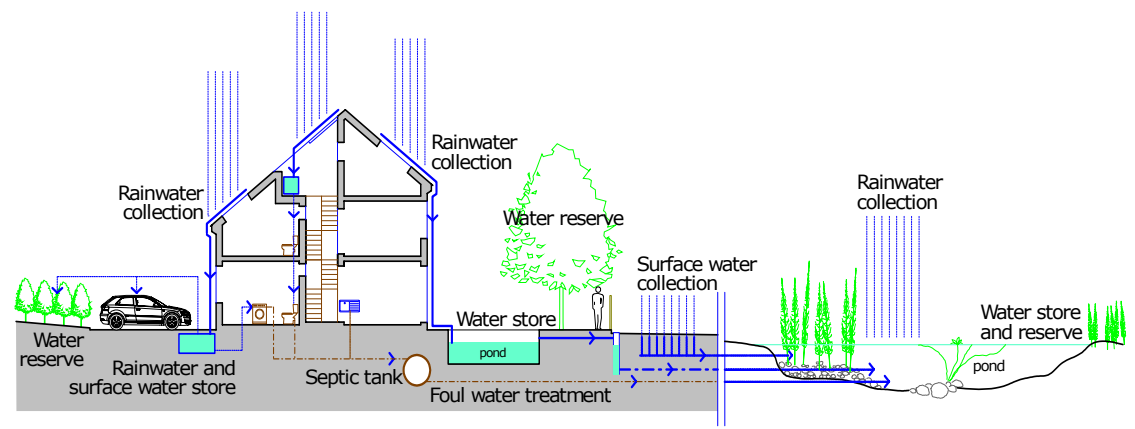

Figure 4: Illustration of water storage, recycling and reuse in residential design. 
supply, rainwater is gathered into a man made ponds from either the sky directly or from the nearby springs or streams. Thus, through the storage of collected water the impact of low water season is minimised, whilst providing for effective cooling in summer when prevailing wind is used to bring the cool air over the water into the house. According to the author's field study observations, almost every village has more than one artificial pond inside a settlement. The recycled water collected in higher pond will be directed into the lower part of the site, and drained into the pond of lower level which recycles water for local agricultural and other production uses. In addition, the principles of collecting rainwater, recycling and reusing 'grey' water are observed in each individual dwelling by using the pond placed in the centre of courtyard.

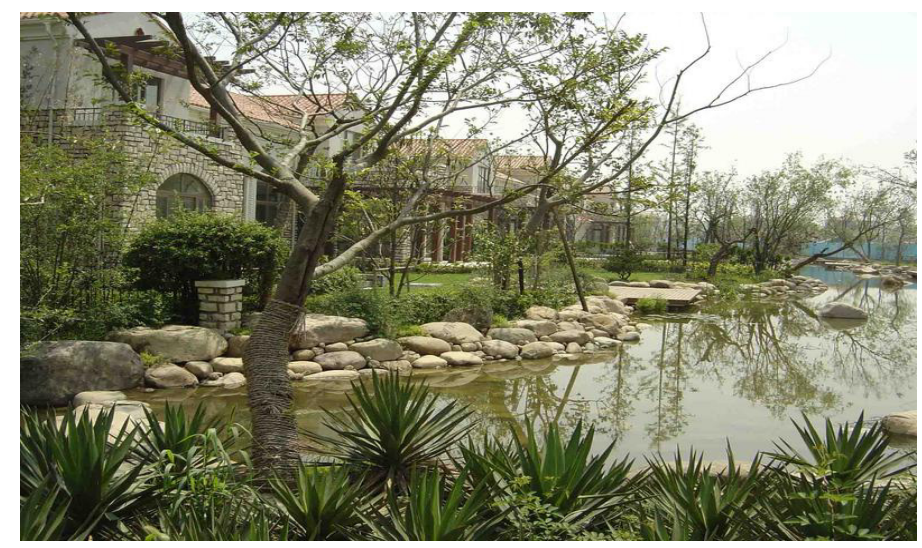

Figure 5: $\quad$ Preservation of an existing natural pond with surrounding artificial landscape in a rural residential area of Shanghai.

\subsection{Landscape, local climate and biodiversity}

FengShui forests are one of the most potent landscape components regarded as a natural heritage in a FengShui settlement [8]. In contemporary residential design, well arranged trees and vegetation are able to supply purified living Qi to the residential area and the inhabitants, whilst absorbing carbon dioxide. Surrounding trees can act as a natural shelter around the edge of a site, creating a physical barrier between inside and outside and delivering the sense of privacy, protection and security in a subtle and natural manner. More importantly, they deter the invasion of detrimental Qi, such as dust and traffic noise from outside, thus reducing the related negative impact (see Figure 6). From the environmental and ecological perspective, well organised green spaces are capable of preventing soil erosion, protecting water resources, improving local climate, providing fresh air, alleviating flood and drought, breaking unfavourable monsoons and sheltering from direct sunshine.

In addition, the connection between newly planted trees and existing forests provides expansive green belts around cities and thus facilitate local biodiversity, growth of different species of vegetations and the movement of animals. 


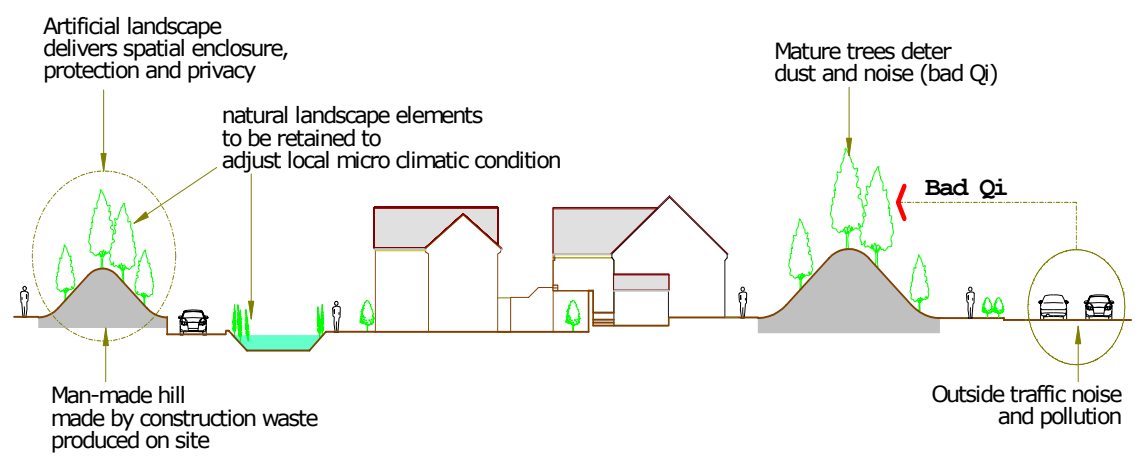

Figure 6: Function of landscape features in a residential area.

China is a large country that covers expansive areas of land. The climate changes distinctively in terms of different regions. Building type in one area might not be suitable for another. Thus, local climate and culture is one of the key factors when deciding on the architectural styles, forms and details in FengShui practice. In China, many representative residential building types demonstrate people's understanding and adjustment to local climate.

\section{Conclusions}

During the recent years, global warming, pollution, consumption of large amount of natural resources and unprecedented economic growth have brought about fundamental changes in the conditions of living environment and attitude of people in urban China. Most cities and places encounter many difficulties such as deterioration of local climate, disappearance of local plants and seasonal animals, acute depletion of energy resources and pollution of water and air.

Living conditions in today's urban Chinese settings have deteriorated and the interrelationship between environment and human behaviour has been severely damaged. The increase in living and working pressures and urbanisation in dense cities have reduced people appreciation of their immediate environment. Moreover, the great demand for accommodation is being catered for by the high rise residential developments, normative floor plan sizing and repetitive architectural layouts that have not only reduced the diversity and creativity in design, but also failed to reflect environmental concerns, social inclusion, individuals, community and their living needs in $21^{\text {st }}$ century.

In addition, the times of post War communist thinking both in terms of social and urban order have long gone. People in China celebrate individuality and have different interests and diverse requirements. Therefore, it is necessary to create living environments that enable people to seek out a family place where they feel treated as individual, comfortable and confident; where they can make sense of the environment, whilst at same time engaging with it. Urban planning and design needs to create opportunities within a context of the site to establish means of communication, integration, inclusion and sense of belonging between inhabitants in local community. Design should further consider balanced use of 
natural energy, recycling and reuse of natural resources, saving energy and living with nature, with increased emphasis on the sustainable residential developments.

Practically, the modern interpretation of FengShui provides creative and inspirational platform for responding to above concerns. FengShui principles, concepts, theories, environmental model and its influences and manifestations have many extensive and adaptive values that can be applied in the sustainable residential development in today's China. For example, the interpretation of the energy movement between Yin and Yang and the energy circulation and transformation between Five Elements provides an inspirational and comprehensive aid for instructing the relationship between energy, materials, humans and information in the architecture of living environment.

In the modern context, FengShui can be practiced as a contemporary philosophy and lifestyle that generates broad modern interpretations and design principles. These can be translated and transformed into a set of design methods that are capable of integrating architecture with environmental and ecological concerns applicable in the pursuance of true harmony and balance between humans, built environment and nature.

\section{References}

[1] Zhong, Z.Q., Ceranic, B. (2007), 'FengShui - A systematic research of vernacular sustainable development in ancient China and its lessons for future', CD-ROM Proc. of the $7^{\text {th }}$ UK CARE Annual General Meeting, eds. J. Kang, H. Xie, H. Liao, Sheffield, UK, 2007.

[2] Huang, Y.S., (2001), 'Traditional Chinese spirit of freedom - a brief exposition of the outlook on freedom embodied in Confucianism, Taoism and Buddhism', Theory Journal, No.3

[3] Zhang, J., (2007), 'Formation of Taoism and the reasons of its decline', Journal of Dali University, No.1

[4] Zhang, X.S., (2003), 'Confucianism and Confucian philosophy', Journal of Oriental Forum, No.5

[5] Shchutskii, L.K. (1979), 'Researches on the Chinese I Ching', New Jersey: Princeton University Press

[6] Yu, K.J. (19980, 'The Ideal Landscapes - the Meanings of FengShui', Beijing: Business Publication

[7] Kaplan, R. \& Kaplan, S. (19890), 'The experience of nature - a psychological perspective', New York: Cambridge University Press

[8] Q. H. Wang (1992), 'The exploration of FengShui in the design of the royal tombs of the Qing Dynasty', Research of FengShui Theory, Tianjin: Tianjin University Press (1992)

[9] Marafa, L. (2003), 'Integrating natural and cultural heritage: the advantage of FengShui landscape resources', International Journal of Heritage Studies

[10] Lip, E. (1979), 'Chinese Geomancy', Times Book International, Singapore 